\title{
REPLACEMENT ION CHROMATOGRAPHY WITH POTENTIO- METRIC DETECTION USING A POTASSIUM-SELECTIVE MEMBRANE ELECTRODE
}

\author{
MAREK TROJANOWICZ \\ Department of Chemistry, University of Warsaw, Pasteura 1, 02-093 Warsaw (Poland)
}

MARK E. MEYERHOFF

Department of Chemistry, University of Michigan, Ann Arbor, MI 48109-1055 (U.S.A.)

(Received 16th September 1988)

\section{SUMMARY}

The use of a simple potassium-selective membrane electrode as a detector in replacement ion chromatography of both cations and anions is examined. For cation chromatography, separated alkali metal ions are detected in the effluent of a suppressed chromatographic system by replacement with potassium ions via cation-exchange tubing bathed in potassium chloride solution. Subsequent downstream potentiometric measurement of the exchanged potassium ions is made with a wall-jet type valinomycin-based polymer membrane electrode. The same replacement stage and potentiometric detector can be used for anion chromatography, in which case counter hydrogen ions in the effluent are the cationic species replaced by potassium. The detection capabilities of the method are shown to depend on a number of factors, including the lengths of suppressor and replacement ion-exchange tubing, the nature and concentration of the bathing electrolytes in the suppressor and replacement stages and the system flow-rates. Under optimized experimental conditions, it is shown that the membrane electrode-based ion replacement scheme can rival the conventional conductivity methods for the detection of separated ions.

High-performance ion chromatography, introduced to modern analytical chemistry in the mid-1970s, is an attractive method for simultaneous multicomponent analysis of mixtures of anions and cations. In most applications of either suppressed or single-column (non-suppressed) systems, conductivity detection is used. However, the high temperature coefficients and limited detection capabilities of the conductimetric method have prompted efforts to implement a variety of novel direct and indirect spectrophotometric and electrochemical detection schemes in ion chromatography [1]. In addition, numerous post-column reaction methods to enhance sensitivity have been proposed [2].

In suppressed-ion chromatography, the high conductivity of the eluent is 
dramatically reduced via a neutralization reaction within packed-bed ion-exchange stripper columns or hollow ion-exchange tubings (bathed in appropriate regenerant solution) placed after the analytical column. Similar ion-exchange processes in a third column or tubing placed in series with the analytical and suppressor stages have become the basis for replacement ion chromatography (RIC), a detection scheme introduced by Hieftje and co-workers [35]. In RIC, effluent from the separation and suppressor columns (or tubing) is pumped through an ion-exchange tube (or column) immersed in an appropriate electrolyte solution containing specific replacement ions. Separated analyte ions or their counter ions are quantitatively exchanged for the replacement ions, which are then monitored downstream with a suitable detector. In the earliest work, lithium was used as the replacement ion in conjunction with flame photometric detection $[3,4]$, whereas more recently nitrate and iodate have served as indicator ions with UV detection [5]. However, the need for more complex and costly detection instrumentation to monitor the replacement ions (beyond the chromatographic equipment) clearly detracts from the fundamental advantages of the replacement concept.

Several types of potentiometric electrodes have already found wide application as detectors in ion chromatography $[1,6]$. These devices have included simple metallic electrodes (e.g., copper), silver electrodes of the second kind and various ion-selective membrane electrodes. In certain instances, e.g., the determination of bromide [7] and ammonium ions [8], the detection limits obtainable via potentiometric detection were better than those possible by any other ion chromatographic detection method.

In this work, the feasibility of using a simple valinomycin-based potassium ion-selective membrane electrode as a universal potentiometric detector in the RIC of cations and anions was examined. As expected based on the electrode's cation selectivity pattern, the use of the potassium sensor alone as a direct detector for separated alkali metal cations provides high sensitivity for potassium, rubidium and cesium ions, but a much poorer response toward ammonium, lithium and sodium [8]. However, it is shown here that utilization of the potassium electrode in a replacement-type detection arrangement (with potassium as the replacement ion) provides a practical means of exploiting the excellent detection capabilities and selectivity of this electrode for monitoring a wide range of ions.

\section{EXPERIMENTAL}

\section{Apparatus}

A schematic diagram of the instrumental setup used for the RIC experiments is shown in Fig. 1. The system consisted of a Spectra-Physics (San Jose, CA) SP 8700 programmable solvent delivery pump, a Rheodyne (Cotati, CA) Model 7125 injection valve and a Wescam (Santa Clara, CA) Model 213 conductivity 


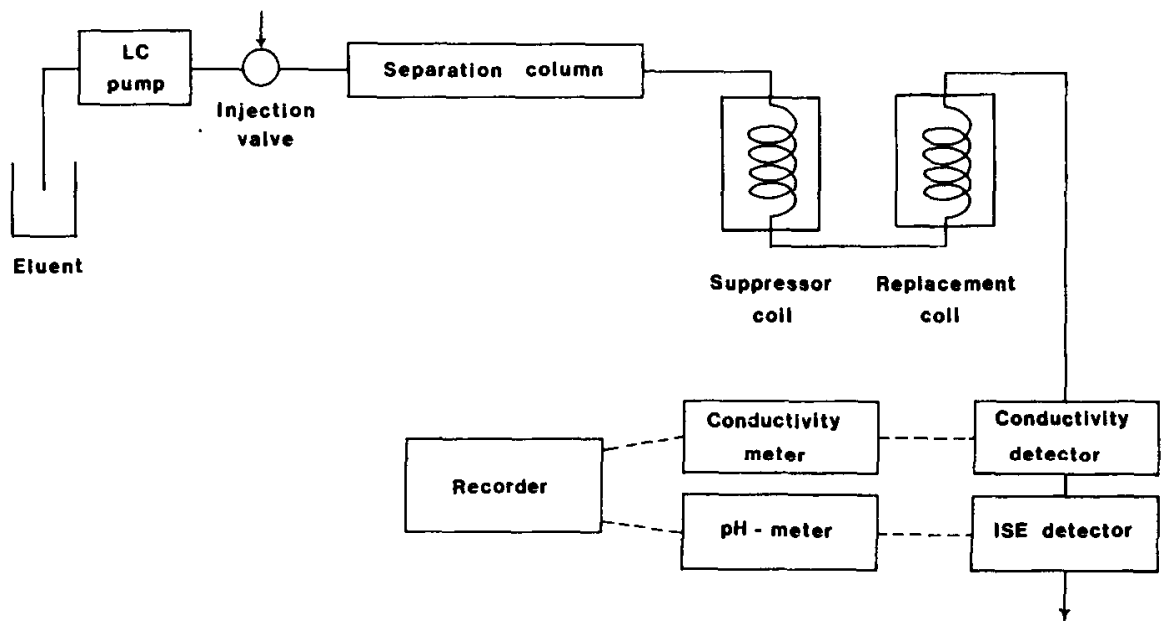

Fig. 1. Schematic diagram of instrumentation used for replacement and conductivity ion chromatographic measurements.

detector. The potentiometric detector was connected to an Accumet Model 910 $\mathrm{pH} / \mathrm{mV}$ meter (Fisher Scientific, Pittsburgh, PA). Analog outputs from both the conductivity and potentiometric detectors were recorded on a Fisher Recordall model D5117-5AQ strip-chart recorder. In several experiments aimed at determining the effect of the LC pump components on the conductivity of the effluents, solutions were pumped through various stages of the system via a Rainin (Woburn, MA) Minipulse multi-channel peristaltic pump.

\section{Columns}

Cations were separated on an ION-210 cation-exchange column (Interaction Chemicals, Mountain View, CA) and anions on a PRP-X100 column (Hamilton, Reno, NV). In both cases, an IonGuard polymeric column (Interaction Chemicals) was placed between the injection valve and the analytical column.

Post-column suppression in anion chromatography was achieved using 30150-cm lengths of Raipore strong cation-exchange tubing (T-1010; RAI Research, Hauppauge, NY) or $250 \mathrm{~cm}$ of Nafion $811 \mathrm{X}$ tubing (Perma Pure Products, Toms River, NJ). The suppressor used for cation chromatography was a $120-\mathrm{cm}$ length of Raipore T-1030 strong anion-exchange tubing. These ionexchange suppressor tubings were coiled $(4 \mathrm{~cm}$ diameter $)$ and placed in 1-1 beakers containing appropriate acid or base solutions. The bathing solutions were stirred with a magnetic stirring bar and changed periodically as determined from observing the baseline detector drift of the entire chromatographic system.

The replacement stage of the RIC system consisted of a coiled $120-\mathrm{cm}$ length 
of Raipore T-1010 cation-exchange tubing bathed in potassium chloride solution.

\section{Potentiometric detector}

Potentiometric detection was done using a large-volume wall-jet electrode design similar to that described previously for solid-state membrane electrodes [9]. A Phillips electrode body (Model IS-561) was fitted with an exchangeable wall-jet cap made of Delrin resin. The outlet nozzle of the cap was $0.56 \mathrm{~mm}$ in diameter and the outlet orifice was positioned $4 \mathrm{~mm}$ from the surface of the potassium-selective membrane mounted in the electrode. The indicating electrode together with a saturated calomel reference electrode were placed in a $100-\mathrm{ml}$ beaker, such that the effluent solution waste was kept $1 \mathrm{~cm}$ above the polymer membrane of the indicating electrode.

The potassium-selective polymer membrane of the indicating electrode was prepared by evaporating a mixture containing $2.0 \mathrm{mg}$ of valinomycin (Sigma, St. Louis, MO), $66 \mathrm{mg}$ of high-molecular weight poly (vinyl chloride) (Fluka, Ronkonkoma, NY), $132 \mathrm{mg}$ of bis (2-ethylhexyl) sebacate (Fluka) and $2 \mathrm{ml}$ of freshly distilled tetrahydrofuran (Aldrich, Milwaukee, WI) from a flat glass surface $\left(3.8 \mathrm{~cm}^{2}\right)$. Smaller pieces of this larger membrane were cut and mounted in the Philips electrode body. A $0.1 \mathrm{M}$ potassium chloride solution was used as the internal reference solution.

\section{Reagents}

Analytical-reagent grade chemicals were used and all solutions were prepared with deionized water obtained from a Milli-Q system (Millipore, Bedford, MA).

Solutions of nitric acid used as eluents in cation chromatography were prepared by diluting concentrated nitric acid (Mallinckrodt, Paris, KY). Sodium hydroxide solutions used as eluents for anion separations and for suppression in cation chromatography were prepared from the solid material (Aldrich). Hydrochloric acid suppressor solutions (for anion chromatography) were prepared by diluting the concentrated acid (Mallinckrodt).

Mixtures of sample cations were prepared from ammonium chloride, lithium chloride, potassium chloride and sodium chloride. Anion mixtures containing fluoride, nitrate, chloride and bromide were prepared with the sodium or potassium salts of these species.

\section{RESULTS AND DISCUSSION}

Flow-injection measurements of potassium

Owing to its high selectivity and reproducibility, long lifetime and low limits of detection (approaching micromolar levels [10]), the potassium-selective electrode using valinomycin in a polymeric membrane has found a wide range 
of analytical applications. Several workers have employed various forms of this electrode in flow-injection arrangements [11-13], where the measurement conditions are similar to those used in ion chromatography. An especially convenient configuration for employing membrane electrodes as flow-through detectors is to use a large-volume wall-jet electrode configuration [9]. This design allows for the use of conventional electrode bodies fitted with exchangeable wall-jet caps. Until now, this design has been used only with solid-state type membrane electrodes $[9,14-17]$. Thus, in preliminary studies of the proposed RIC system, flow-injection studies were carried out to determine whether the large-volume wall-jet electrode design could be used in conjunction with the polymer membrane-type potassium electrode.

Flow-injection measurements were made using $0.1 \mathrm{M}$ sodium chloride as a carrier solution and a 100- $\mu$ injection loop. Peaks recorded for potassium concentrations ranging from $10 \mu \mathrm{M}$ to $1 \mathrm{mM}$ at a flow-rate of $1 \mathrm{ml} \mathrm{min}^{-1}$ showed reasonable reproducibility (see Fig. 2; s.d. of $\Delta E$ values $< \pm 0.5 \mathrm{mV}$ for $n=5$ ). Peak potentials recorded with the guard column in place (between the injector and the electrode detector, Fig. 2A) were not significantly different than those obtained without the guard column (Fig. 2B). A near-Nernstian relationship between the logarithm of injected potassium concentrations and peak height (in $\mathrm{mV}$ ) was observed for peaks greater than $50 \mathrm{mV}$ for both flowing configurations. Below this limit, the calibration plot ( $\Delta E$ vs. $\log \mathrm{K}^{+}$concentration)

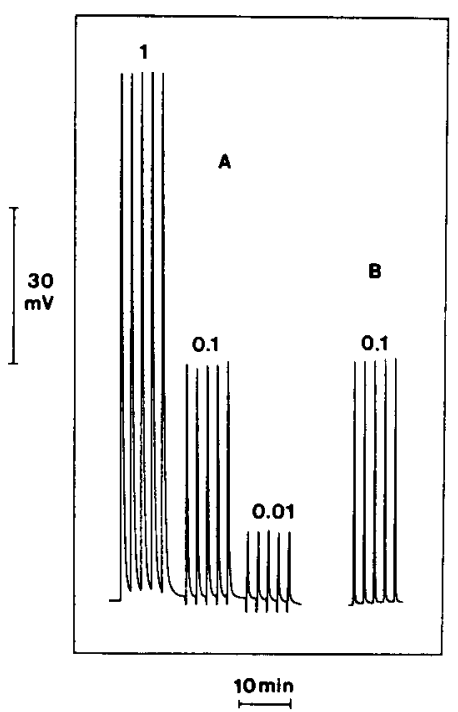

Fig. 2. Flow-injection peaks recorded using potassium-selective electrode in the chromatographic setup without a separation column and (A) with and (B) without a guard column. Carrier solu-

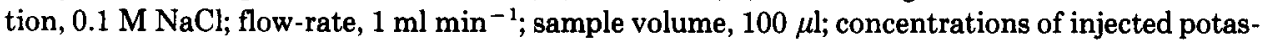
sium chloride solutions, $0.01-1 \mathrm{mM}$ as shown. 
exhibited a non-linear response. This observation correlates well with previous results obtained for flow-injection measurements with solid-state membrane electrodes. [18]. The selectivity of the flow-through electrode used here for potassium over other metal ions was similar to that observed for other valinomycin-PVC membranes [19].

\section{RIC with potentiometric potassium detection}

The general reaction sequences involved in RIC have been described in detail by Galante and Hieftje [4,5]. Since the ultimate detection capabilities of the present system depend on the background concentration of cations in the effluent (due to the replacement of all cations by potassium), chromatography of both cations and anions must be done using a suppressed system. Ideal suppression in cation chromatography results from the exchange of hydroxyl ions for the anions of a strong eluent acid to yield a water background. Thus, separated cations elute with hydroxyl counter ions. In the replacement stage, potassium ions exchange for each of the separated cations in the effluent. Any trace contamination of cations in the eluent will cause an increase in the background potentiometric potassium signal. The major sources of such background contamination in RIC have been detailed previously [4,5]. As shown in Table 1, these sources of contamination also contribute to the background total ion content of the effluent, as measured by a conductivity detector. It can be seen that the addition of the suppressor tubing to the total flow system causes a dramatic increase in the background conductivity, and that variations

TABLE 1

Background conductivity measured for various flow system configurations

\begin{tabular}{|c|c|c|c|c|}
\hline $\begin{array}{l}\text { Configuration of } \\
\text { pumping system }\end{array}$ & Solution pumped & $\begin{array}{l}\text { Solution in } \\
\text { suppressor } \\
\text { reservoir }\end{array}$ & $\begin{array}{l}\text { Flow-rate } \\
\left(\mathrm{ml} \mathrm{min}^{-1}\right)\end{array}$ & Conductivity $(\mu \mathrm{S})$ \\
\hline $\begin{array}{l}\text { Peristaltic pump with } \\
\text { Tygon tubing }\end{array}$ & Deionized water & - & $\begin{array}{l}0.4 \\
0.8 \\
3.0\end{array}$ & $\begin{array}{l}0.024 \\
0.017 \\
0.014\end{array}$ \\
\hline $\begin{array}{l}\text { Ion chromatography } \\
\text { with guard column }\end{array}$ & Deionized water & - & 3.0 & 0.048 \\
\hline $\begin{array}{l}\text { Ion chromatography } \\
\text { with } 90-\mathrm{cm} \text { Raipore } \\
\text { suppressor tubing }\end{array}$ & $10 \mathrm{mM} \mathrm{HNO}_{3}$ & $10 \mathrm{mM} \mathrm{NaOH}$ & $\begin{array}{l}0.4 \\
0.8 \\
1.2\end{array}$ & $\begin{array}{l}0.296(8.9)^{b} \\
0.137(7.9)^{b} \\
1.08(4.2)^{b}\end{array}$ \\
\hline $\begin{array}{l}\text { Ion chromatography } \\
\text { with } 150-\mathrm{cm} \text { Raipore } \\
\text { suppressor tubing }\end{array}$ & $\begin{array}{l}10 \mathrm{mM} \mathrm{HNO}_{3} \\
10 \mathrm{mM} \mathrm{HNO}_{3}\end{array}$ & $\begin{array}{r}10 \mathrm{mM} \mathrm{NaOH} \\
1 \mathrm{mM} \mathrm{NaOH}\end{array}$ & 0.8 & 0.104 \\
\hline
\end{tabular}

${ }^{a}$ Anion-exchange tubing. ${ }^{\mathrm{b}} \mathrm{pH}$ of effluent in parentheses. 
in flow through this tubing have a marked effect on the total ion concentration of the background effluent (due to kinetics of exchange process in suppressor tubing). Decreasing the bathing $\mathrm{NaOH}$ concentration in the suppressor stage reduces the observed effluent conductivity, but at the expense of requiring more frequent changes of this solution.

For anion chromatography, sodium hydroxide may be used as the eluent and suppression achieved via exchange of sodium for hydrogens ions in the suppressor tubing. Consequently, separated anions elute as acids and potentiometric detection with the potassium electrode is based on exchange of the counter hydrogen ions for potassium ions. The detection capabilities in this case are again limited by the same sources of background ions suggested by Galante and Hieftje [4,5].

\section{Optimization of RIC for cations}

The quality of results obtained in RIC depends on optimizing the conditions used for suppression and ion replacement following suppression. Adjustment of the experimental conditions can be controlled by monitoring the $\mathrm{pH}$ of the effluent after suppression, the conductivity after the suppression and replacement coils and the final potentiometric signal recorded with the potassium electrode. Chromatograms for a mixture of lithium, sodium, ammonium and potassium ions using $10 \mathrm{mM}$ nitric acid as the eluent, recorded under various suppression and replacement conditions, are shown in Fig. 3. Potentiometric detection with the wall-jet potassium electrode placed immediately after the anion-exchange suppressor (i.e., no replacement stage) results in large signals for separated potassium, but much smaller responses toward the other cations (Fig. 3A). Addition of a short cation-exchange replacement tubing $(30 \mathrm{~cm})$ bathed in $0.02 \mathrm{mM} \mathrm{KCl}$ immediately before the electrode results in a decrease in the potassium and ammonium peaks and an increase in the sodium and lithium signals (Fig. 3B). A longer replacement coil $(90 \mathrm{~cm})$ coupled with an increase in the replacement bathing solution to $1 \mathrm{mM}$ (Fig. 3C) yields even larger signals for lithium and sodium (more replacement) and smaller net signals for potassium owing to increased dispersion. The decrease in the magnitude of the ammonium ion signal (in Fig. 3C) results from loss of neutral ammonia through the walls of the extended length of replacement tubing owing to the fact that the effluent is basic (see Table 1 and discussion below).

It should be noted that the baseline potential of the potassium electrode under optimized conditions (Fig. $3 \mathrm{C}$ ) corresponds to a background potassium level of roughly $10 \mu \mathrm{M}$ in the effluent. Increasing the bathing potassium chloride level $(>1 \mathrm{mM})$ in the replacement stage results in an increase in this background signal and thus smaller peaks. This is due to a finite leakage of counter chloride ions through the cation-exchange replacement tubing. Similarly, leakage of sodium counter ions through the walls of the anion-exchange suppressor tubing limits the concentration of bathing $\mathrm{NaOH}$ solution to 10 


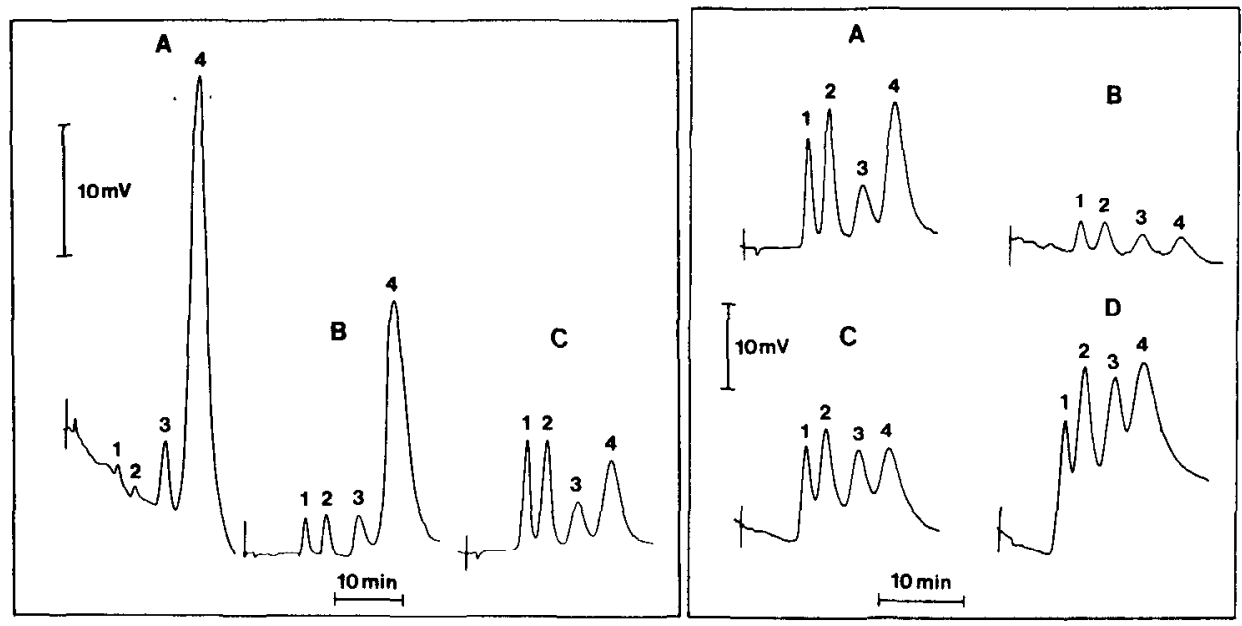

Fig. 3. Cation chromatograms obtained in (A) suppressed and (B, C) replacement systems with $10 \mathrm{mM}$ nitric acid as eluent at a flow-rate of $0.8 \mathrm{ml} \mathrm{min}^{-1}$ using potentiometric detection via the potassium-selective electrode. Concentration of $\mathrm{NaOH}$ used as regenerant in suppressor: (A) 1 $\mathrm{mM}$; (B, C) $10 \mathrm{mM}$. Concentration of $\mathrm{KCl}$ used for replacement: (B) $0.02 \mathrm{mM}$; (C) $1 \mathrm{mM}$. Replacement, Raipore tubing: (B) $30 \mathrm{~cm}$ and (C) $90 \mathrm{~cm}$. Peaks: $1=\mathrm{Li}^{+} ; 2=\mathrm{Na}^{+} ; 3=\mathrm{NH}_{4}{ }^{+}$; $4=\mathrm{K}^{+}$. Injection: $10 \mu \mathrm{l}$ of a $10 \mathrm{mM}$ solution of each cation.

Fig. 4. Chromatograms obtained in RIC of cations using $10 \mathrm{mM}$ nitric acid as eluent at a flow-rate of $0.8 \mathrm{ml} \mathrm{min}^{-1}$ via potentiometric detection with the potassium-selective electrode. Regenerants for suppressor: (A, C) $10 \mathrm{mM} \mathrm{NaOH}$; (B) $10 \mathrm{mM}$ sodium borate; (D) $5 \mathrm{mM} \mathrm{Ba}(\mathrm{OH})_{2}$. Regenerants for replacement: (A, B, D) $1 \mathrm{mM} \mathrm{KCl}$; (C) $0.5 \mathrm{mM} \mathrm{K}_{2} \mathrm{HPO}_{4}$. Peaks: $1=\mathrm{Li}^{+} ; 2=\mathrm{Na}^{+}$; $3=\mathrm{NH}_{4}{ }^{+} ; 4=\mathrm{K}^{+}$. Injection: $10 \mu \mathrm{l}$ of a $10 \mathrm{mM}$ solution of each cation. (Note: baseline potential in $\mathrm{B}$ is ca. $45 \mathrm{mV}$ more positive than in $\mathrm{A}, \mathrm{C}$ and $\mathrm{D}$, indicating a higher background of potassium ions in the effluent; see text.)

$\mathrm{mM}$. Such leakage of counter ions is due to breakdown of the Donnan potential when the gradient of cations or anions across the walls of the replacement and suppressor tubings, respectively, is high (e.g., when effluent is fully suppressed to nearly a pure water background). Under these conditions, the effluent from the suppressor stage is still fairly basic (e.g., $\mathrm{pH}$ ca. 9.0) and this, in part, controls the ultimate detection capabilities of the present system.

The specific electrolyte solutions used to bathe the suppressor and replacement tubings in the RIC system also influence the results obtained (Fig. 4). For example, use of sodium borate in place of sodium hydroxide in the suppressor reservoir produces much smaller potentiometric signals (Fig. 4B) owing to an increase (from 10 to ca. $70 \mu \mathrm{M}$ ) in the background potassium levels in the final effluent (i.e, less effective suppression). The use of barium hydroxide causes a severe resolution problem (Fig. 4D). Similar resolution problems were encountered when potassium chloride in the replacement reservoir was changed to dipotassium hydrogenphosphate (Fig. 4C). These findings are 
in agreement with observations reported by Galante and Hieftje [4,5], who suggested that the kinetics of the replacement and suppression processes can be influenced by the nature of the bathing electrolyte in each portion of the system, and these effects can have a profound impact on the detection capabilities and resolution of the final RIC system.

For the given chromatographic system used in this work (e.g., column, suppressor, eluent), experimental detection limits using conductivity are poorer than those reported by others for suppressed chromatography of monovalent cations [1]. However, since some of the same system factors which limit ion sensitivity using conductivity detection also influence the results obtainable with the replacement approach, for a particular experimental setup, it seems reasonable to evaluate the potentiometric RIC method by directly comparing the results with those obtained with conductivity under the exact same experimental conditions. Figure 5 shows the chromatograms obtained for the separation of cations using suppressed and non-suppressed conductivity detection.

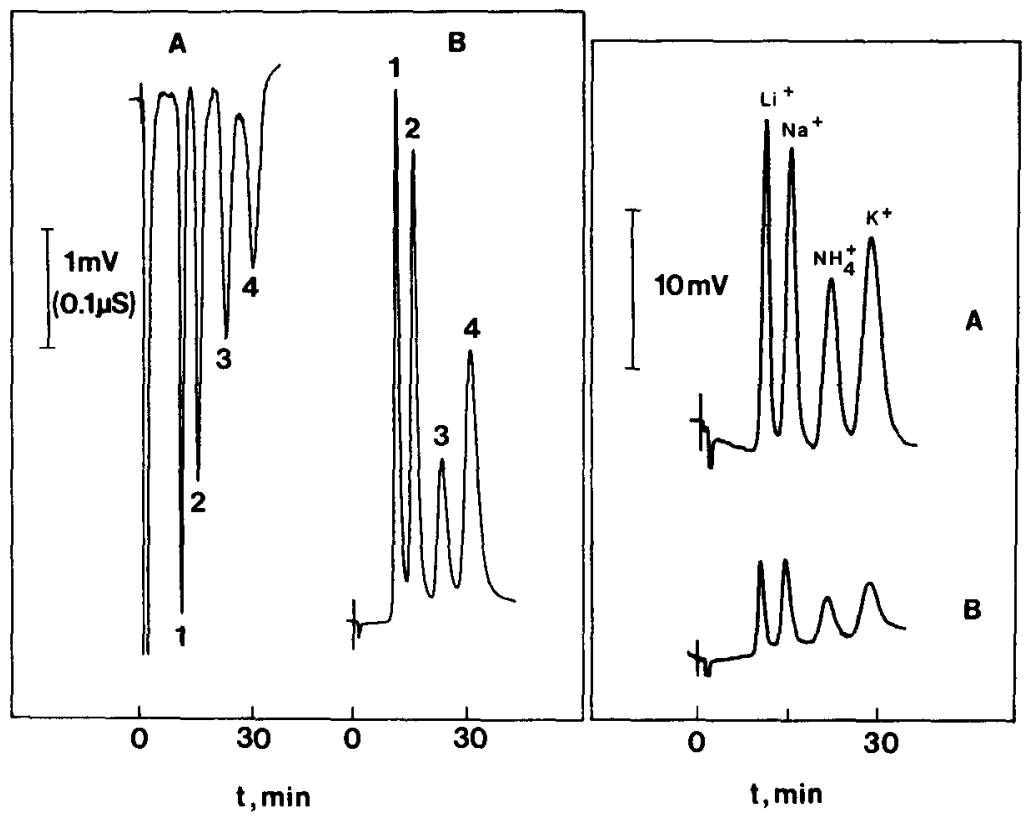

Fig. 5. Chromatograms obtained in (A) non-suppressed and (B) suppressed system with conduc-

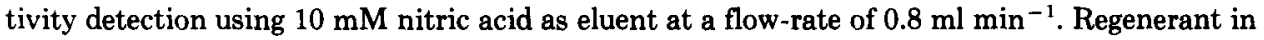
suppressor: $10 \mathrm{mM} \mathrm{NaOH}$. Injection: $100 \mu \mathrm{l}$ of $1 \mathrm{mM}$ (1) $\mathrm{Li}^{+}$, (2) $\mathrm{Na}^{+}$, (3) $\mathrm{NH}_{4}^{+}$and (4) $\mathrm{K}^{+}$.

Fig. 6. Cation chromatograms obtained in replacement system with potentiometric detection using the potassium ion-selective electrode and $10 \mathrm{mM}$ nitric acid as eluent at a flow-rate of $0.8 \mathrm{ml}$ $\mathrm{min}^{-1} .10 \mathrm{mM} \mathrm{NaOH}$ used as regenerant for suppressor and $1 \mathrm{mM} \mathrm{KCl}$ as regenerant for replacement. Injection: $100 \mu \mathrm{l}$ of (1) $1 \mathrm{mM}$ and (B) $0.2 \mathrm{mM}$ of each cation. Relative peak areas for A: $1.0: 1.3 ; 1.0: 1.7$ (via manual integration). 
Despite the similar magnitude of the signals with both versions of conductivity detection, the non-suppressed conditions (single column) are much less favorable owing to the higher background noise in the highly conductive eluent signal (e.g., $110 \mu \mathrm{S}$ ). Detection limits with the suppressed conductivity detection scheme were estimated to be $9,11,35$ and $21 \mu \mathrm{M}$ for $\mathrm{Li}^{+}, \mathrm{Na}^{+}, \mathrm{NH}_{4}{ }^{+}$and $\mathrm{K}^{+}$, respectively (using $S / N=3$ ). Figure 6 shows the results obtained under the same experimental conditions using the RIC approach with the potassium electrode as the detector. A noise level of $0.1 \mathrm{mV}$ resulted in detection limits of $10,10,21$ and $17 \mu \mathrm{M}$ for $\mathrm{Li}^{+}, \mathrm{Na}^{+}, \mathrm{NH}_{4}{ }^{+}$and $\mathrm{K}^{+}$, respectively, indicating nearly equal sensitivity to the suppressed conductivity method. With the use of improved signal processing instrumentation (e.g., high input impedance amplifiers ) and pulse suppression methods to reduce background noise, it seems likely that even better detection limits should be attainable with the membrane electrode RIC system.

Calibration graphs (peak height vs. injected concentration) for the separated cations using potentiometric detection are shown in Fig. 7. In general, the graphs are linear up to potential changes approaching $20 \mathrm{mV}$, after which the logarithmic nature of the detector predominates. The high intercept for the sodium graph is probably due to trace levels of sodium in each of the cation
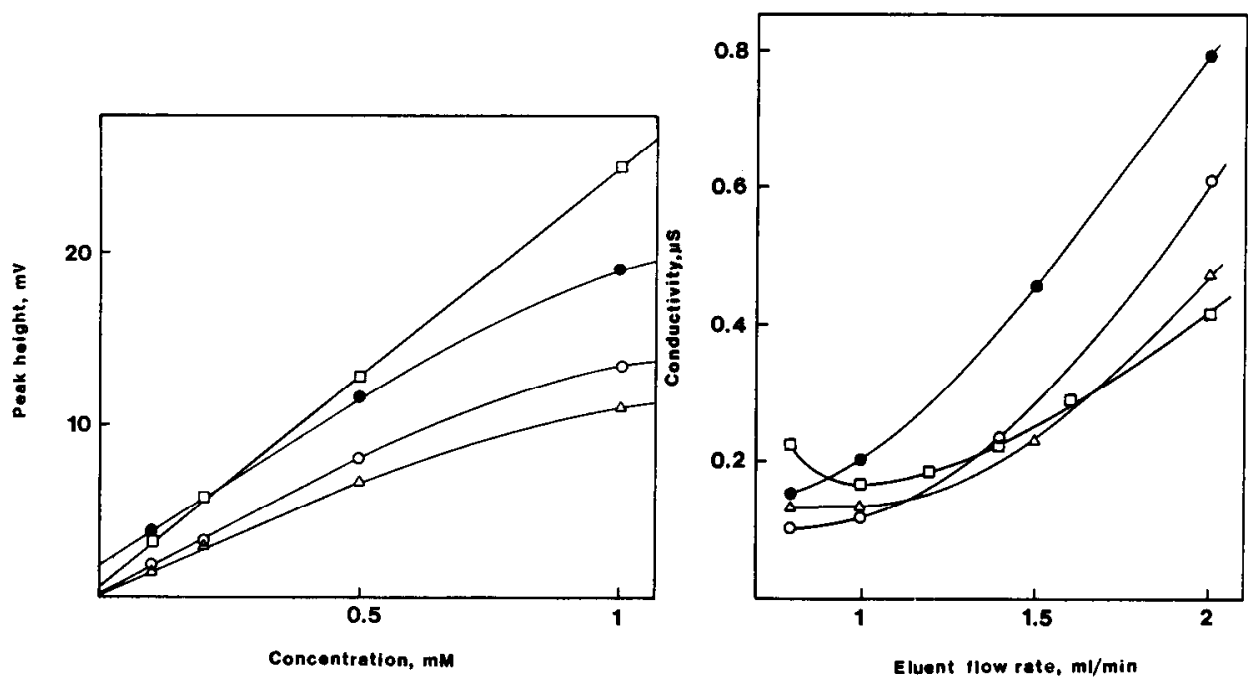

Fig. 7. Calibration graphs obtained for $(\square) \mathrm{Li}^{+},(\bigcirc) \mathrm{Na}^{+},(\Delta) \mathrm{NH}_{4}{ }^{+}$and $(\mathrm{O}) \mathrm{K}^{+}$in RIC using potentiometric detection with the potassium membrane-selective electrode. Conditions as in Fig. 6.

Fig. 8. Effect of regenerant concentration in suppressor on conductivity of effluent in ion chromatography of anions using $15 \mathrm{mM} \mathrm{NaOH}$ as eluent and 250-cm Nafion $811 \mathrm{X}$ tubing as suppressor. Regenerant: (O) $2 \mathrm{mM} \mathrm{HCl}$; $\triangle$ ) $4 \mathrm{mM} \mathrm{HCl}$; ( $\square$ ) $12 \mathrm{mM} \mathrm{HCl}$; (O) $6 \mathrm{mM} \mathrm{H}_{2} \mathrm{SO}_{4}$. 
salts used to prepare the mixture. As with all other detectors used with RIC, the potassium electrode detector yields smaller differences in the sensitivity toward the various separated ions than conductivity detection, particularly if the integrals of the peaks are used (see legend to Fig. 6). However, it should be noted that, since the rates of ion exchange for various sample ions can differ, the ion replacement methods based on tubular replacement stages can rarely offer equal detection capabilities (in terms of peak areas) toward all separated ions (as suggested by Galante and Hieftje [4]) unless lengthy and packed replacement stages are used.

\section{Optimization of RIC for anions}

The Hamilton PRP-X100 column is designed for performing anion chromatography over $\mathrm{pH}$ range 1-13 [20]. Thus, dilute sodium hydroxide was found to be a suitable eluent for the separation of a model mixture of fluoride, chloride, bromide and nitrite using flow-rates of 1-2 $\mathrm{ml} \mathrm{min}^{-1}$.

Suppression in the anion chromatography systems was achieved with various cation-exchange tubings using nitric, hydrochloric or sulfuric acid as the bathing regenerant solution. To gauge the effectiveness of the different acids and their concentrations at suppressing the sodium hydroxide eluent, conductivity detection was employed after the suppressor tubing, at various flow-rates of the eluent (using a 250-cm length of Nafion 811-X tubing). As shown in Fig. 8, the lowest conductivity was found with $2 \mathrm{mM}$ hydrochloric acid as the bathing solution at flow-rates approaching $1 \mathrm{ml} \mathrm{min}-1$. However, such dilute acid is rapidly consumed and therefore, for practical purposes, more concentrated hydrochloric acid ( $12 \mathrm{mM}$ ) was used for subsequent experiments. Both sulfuric acid (example shown in Fig. 8) and nitric acid (not shown) were much less effective at suppressing the conductivity of the effluent.

Similar studies were conducted with the replacement stage of the system by varying the concentration of bathing $\mathrm{KCl}$ from 0.5 to $6 \mathrm{mM}$. The lowest conductivity $(0.16 \mu \mathrm{S})$ after the suppressor and replacement stages was obtained using $2 \mathrm{mM} \mathrm{KCl}$ as the replacement electrolyte and a flow-rate of $2 \mathrm{ml} \mathrm{min}^{-1}$. The conductivity of the effluent doubled when the flow-rate was reduced to 1 $\mathrm{ml} \mathrm{min}{ }^{-1}$. The potentials of the wall-jet potassium electrode under such conditions were nearly the same as observed for the cation RIC system (e.g., ca. $10 \mu \mathrm{M} \mathrm{K}^{+}$in the effluent).

Results from the preliminary optimization studies under conditions necessary for anion separation suggested that better performance with conductivity detection (when placed after the suppressor, as in conventional suppressedion chromatography) can be expected at lower flow-rates, whereas higher flowrates provide the lowest potassium background for the electrode-based RIC system. Therefore, a direct comparison of both detection arrangements was carried out using $1.0 \mathrm{ml} \mathrm{min}^{-1}$ for conductivity and $2.0 \mathrm{ml} \mathrm{min}^{-1}$ for potentiometric detection with the potassium electrode. Typical anion chromatograms 


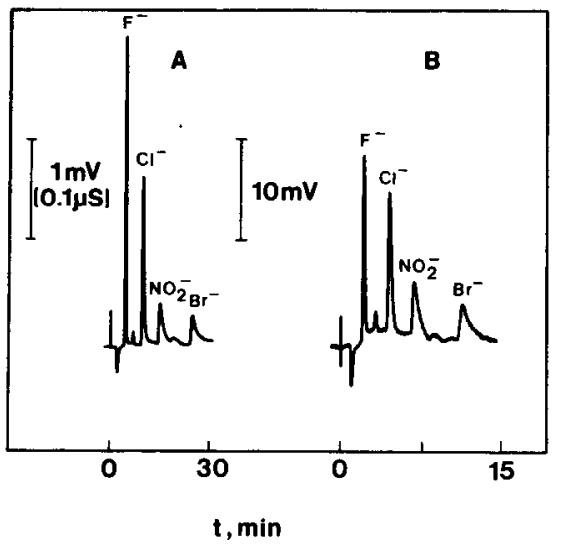

Fig. 9. Anion chromatograms obtained (A) in suppressed systems with conductivity detection and $(B)$ in replacement system with potentiometric detection using the potassium-selective membrane electrode and $15 \mathrm{mM} \mathrm{NaOH}$ as eluent. Flow-rate: (A) 1 and (B) $2 \mathrm{ml} \mathrm{min}^{-1} .12 \mathrm{mM} \mathrm{HCl}$ used as regenerant for suppressor and $2 \mathrm{mM} \mathrm{KCl}$ for replacement. Injection: $100 \mu \mathrm{l}$ of a $0.2 \mathrm{mM}$ solution of each anion. Relative peak areas in $B: 1.0: 1.4: 1.3: 1.2$ (via manual integration).

for both types of detection schemes under these conditions are shown in Fig. 9. Taking into account the background noise level for both methods, detection limits for fluoride, chloride, nitrite and bromide were $2.9,5.3,10$ and $28 \mu \mathrm{M}$, respectively, with conductivity detection, while the electrode-based RIC system yielded detection limits of $3.2,4.3,11$ and $17 \mu \mathrm{M}$, respectively (based on $S / N=3$ ). Based on these results, it appears that the dispersion variations arising from differing flow-rates and the extra length of post-column replacement tubing in the RIC system tend to offset each other, yielding comparable detection limits for the two methods. Anion calibration graphs for the potentiometric method were linear up to peak heights of ca. $15 \mathrm{mV}$ (not shown).

\section{Conclusions}

The results demonstrate that the valinomycin-based potassium membrane electrode can be used effectively as a detector in RIC of cations and anions. Although this study was restricted to the separation and detection of only monovalent inorganic ions, the approach suggested should also be applicable to multivalent and organic ion species. At present, the detection limits are comparable to those obtained using conductivity detection, although further improvements should be possible by decreasing the noise level in the potentiometric detection system and eliminating the sources of background cation contamination and leakage into the effluent flowing from the suppressor and replacement stages of the system. In addition, improvements in the quality of the ion separations and detection should be possible if band dispersion in the lengthy suppression and replacement stages is minimized. This can be accom- 
plished by reducing the inner diameter of the fibers, using inner packing materials to reduce the dead volumes or employing commercial Dionex suppression systems. In view of the simplicity, low cost and accessibility of the required potentiometric equipment, it seems likely that the membrane electrode detector system described here can offer distinct advantages over the various spectroscopic methods used previously in conjunction with RIC.

The authors thank Mr. Michael Collison for preparing the potassium-selective membrane. M. T. expresses his gratitude to Mallinckrodt Sensor Systems for supporting his stay at the University of Michigan.

\section{REFERENCES}

1 P.R. Haddad and P. Jandik, in J.G. Tarter (Ed.), Ion Chromatography, Dekker, New York, 1987, pp. 87-151.

2 I.S. Krull (Ed.), Reaction Detection in Liquid Chromatography, Dekker, New York, 1986.

3 S.W. Downey and G.M. Hieftje, Anal. Chim. Acta, 143 (1983) 1.

4 L.J. Galante and G.M. Hieftje, Anal. Chem., 59 (1987) 2293.

5 L.J. Galante and G.M. Hieftje, Anal. Chem., 60 (1988) 995.

6 M. Trojanowicz, in A. Izaska (Ed.), Proceedings of ElectroFinn Analysis Conference, Turku, Finland, June, 1988, Plenum, New York, in press.

7 J. Slavina, F.P. Bakker, P.A.C. Jongejan, L. van Lamoen and J.J. Mols, Anal. Chim. Acta, 130 (1981) 1.

8 K. Suzuki, M. Aruga and T. Shirou, Anal. Chem., 55 (1983) 2011.

9 L. Ilcheva, M. Trojanowicz and T. Krawczyviski vel Krawczyk, Fresenius' Z. Anal. Chem., 328 (1987) 27.

10 M.E. Collison, unpublished data.

11 J. Ruzicka, E.H. Hansen and E.A.G. Zagatto, Anal. Chim. Acta, 88 (1977) 1.

12 M. Trojanowicz, Z. Augustowska, W. Matuszewski, G. Moraczewska and A. Hulanicki, Talanta, 29 (1982) 113.

13 M.E. Meyerhoff and P.M. Kovach, J. Chem. Educ., 50 (1983) 766.

14 W. Frenzel and P. Brätter, Anal. Chim. Acta, 185 (1986) 127.

15 W. Frenzel and P. Brätter; Anal. Chim. Acta, 187 (1986) 1.

16 W. Frenzel and P. Brätter, Anal. Chim. Acta, 188 (1986) 151.

17 M. Trojanowicz and W. Frenzel, Fresenius' Z. Anal. Chem., 328 (1987) 65.

18 M. Trojanowicz and W. Matuzewski, Anal. Chim. Acta, 138 (1982) 71.

19 K. Umezawa and Y. Umezawa, Selectivity Coefficients for Ion-Selective Electrodes, University of Tokyo Press, Tokyo, 1983.

20 M. Benning, Am. Lab., May (1988) 74. 